\title{
Cultural, Morphological and Molecular Variability of Fusarium oxysporum f. sp. udum Isolates by RAPD Method
}

\author{
P.H. Ghante*, K.M. Kanase, S.P. Kale, R.L. Chavan, K.M. Sharma and S.B. Ghuge \\ Department of Plant Pathology, College of Agriculture, Parbhani, Vasantrao Naik \\ Marathwada Krishi Vidyapeeth, Parbhani 431402 (M.S), India \\ *Corresponding author
}

\section{Keywords}

Pigeonpea wilt, Fusarium oxysporum f. sp. udum, in vivo, Cultural,

morphological and

molecular characteristics

Article Info

Accepted:

15 September 2018

Available Online:

10 October 2018

\section{A B S T R A C T}

Cultural, morphological and molecular characteristics of Fusarium oxysporum f. sp. udum were studied where eight isolates indicated a great variability amongst them. However, the isolate FOC-2 (Jalna) exhibited maximum mycelial growth of $90 \mathrm{~mm}$. The isolates viz., Jalna (FOC-2) and Beed (FOC-3) produced partially submerged (FOC-2) to submerged (FOC-3) white sparse dense growth with smooth margin and bright white substrate pigmentation, respectively. Maximum micro-conidial, macro-conidial and chlamydospore size $(17.20 \mu \mathrm{m}, 30.50 \times 7.00 \mu \mathrm{m}$ and $21.80 \times 19.60 \mu \mathrm{m})$ were recorded in isolate Jalna (FOC-2). The micro-conidia were more or less oval to cylindrical with no septation. The macro-conidia were typically sickle shaped curved, fusoid varied in the size and number of septation (3-5). The chlamydospores were round to oval in shape. Genetic diversity was analyzed based on data obtained by 10 RAPD primers. Most of the primers were found 91.66 to 100 per cent polymorphic in nature. All primers had amplified total number of 144 bands among which 140 and 4 were found polymorphic and monomorphic, respectively. The cluster I comprised isolates FOC-1 (Aurangabad) and FOC-6 (Nanded) together and showed 57.60 per cent similarity to each other; however, cluster II comprised six isolates [FOC-2 (Jalna), FOC-3 (Beed), FOC-4 (Osmanabad), FOC-5 (Latur), FOC-7 (Parbhani) and FOC-8 (Hingoli)] together showing 53.88 per cent similarity. All of these six isolates of cluster II were from different region showing maximum similarity in the range of 59.00 to 100 per cent.

\section{Introduction}

Pigeonpea [Cajanus cajan (L.) Millspaugh] is known by more than 350 vernacular names, the most popular being arhar, yellow dhal, red gram, tur (India), congo pea, gandul, guandu (Brazil), angola pea (United Kingdom), catjang pea, ambrevade, pois d'angdie (French-speaking West Africa), quinochoncho (Venezuela). Pigeonpea ranks fourth in importance as edible legume in the world. Pigeon pea is extensively grown throughout the tropics, subtropics and warmer equatorial regions of Asia, East Africa and Central America in lower altitude areas between $30^{\circ} \mathrm{N}$ to $30^{\circ} \mathrm{S}$, particularly in the semi-arid and lower humid tropics.

Globally, it is grown on approximately 5 million hectares in about 82 countries of the 
world. The major production area is located in India, Myanmar, Kenya, Malawi, Uganda and Tanzania.

The pigeonpea is the first seed legume plant to have its complete genome sequenced. The sequencing was first accomplished by a group of 31 Indian scientists from the Indian Council of Agricultural Research, New Delhi (India). India alone occupies three-fourth of the global harvested area and contributes almost a similar share in production. Pigeonpea occupies a prominent place in Indian rainfed agriculture. It is the second most important pulse crop next to chickpea, covering an area of around 4.42 $\mathrm{m}$ ha (occupying about $14.5 \%$ of area under pulses), production of 2.86 MT (contributing to $16 \%$ of total pulse production) and productivity of about $707 \mathrm{~kg} / \mathrm{ha}$. Deep roots improve physical properties of the soil and pulverize the soil. The plants shed large amount of leaves, this biomass adds organic matter to soil. Besides, it also leaves $30-50 \mathrm{~kg}$ ' $\mathrm{N}$ ' to the succeeding crop and also benefiting the inter-cropped cereals through increased ' $\mathrm{N}$ ' supply. Pigeonpea in some areas is an important crop for green manure, providing up to $90 \mathrm{~kg}$ nitrogen per hectare.

The area of pigeonpea in Maharashtra is increased from 10.39 lakh ha to 15.33 lakh ha in 2016-17. Area of pigeonpea was highest in 2016-17 (15.33 lakh ha) while the production and productivity were highest during 2013-14 i.e.10.34 lakh tones and $906 \mathrm{~kg} / \mathrm{ha}$, respectively. In 2016-17 estimated production of pigeonpea in Maharashtra is 11.70 lakh tonnes. In Marathwada, area under pigeonpea was 5.95 lakh ha during 2016-17, while production and productivity were highest during 2013-14 i.e. 5.16 lakh tones and 933 $\mathrm{kg} / \mathrm{ha}$, respectively (Anonymous 2017). Maharashtra contributes $30.29 \%$ in terms of area with $28.29 \%$ of production at national level (average of last ten years). Percentage of area increase during 2016-17 as compared to previous year (2015-16) is $27.25 \%, 32.22 \%$ and $33.64 \%$ in India, Maharashtra and Marathwada, respectively. In general, there is low productivity of pulses including pigeonpea. Because, the crop is grown on marginal lands, low rainfall areas, poor management, poor crop husbandry, high rate of flower and fruit drop, non- uniform maturity, pod shattering and susceptibility to pests and diseases.

Wilt caused by Fusarium udum is the most destructive disease of pigeonpea throughout India. The plant mortality up to 50 per cent has been observed with severe infection of wilt. The main symptoms are wilting of seedlings and adult plants. The wilting starts gradually showing yellowing and drying of leaves followed by wilting of whole infected plant. The affected plants can easily be recognized in patches in the field. Wilt appears on the young seedlings but mainly observed during flowering and podding stage. Surveys conducted for the disease by Kannaiyan et al., (1984) have indicated it to be a major problem in the states of Bihar and Maharashtra (Reddy et al., 1990). Fusarium wilt characterized by wilting of the affected plants and characteristic internal browning or blackening of the xylem vessels extending from root system to stems. Partial wilting of the plants (Upadhyay and Rai, 1992) and patches of dead plants (Reddy et al., 1993) were reported to be common in the fields during advanced stages of plant growth. Investigation was carried out to study cultural, morphological and molecular variability of test pathogen.

\section{Materials and Methods}

The experiment was conducted at Department of Plant Pathology, College of Agriculture Parbhani, VNMKV, Parbhani (M.S.). The pathogen was isolated from diseased leaves of Pigeonpea on PDA incubated at $27 \pm 2{ }^{\circ} \mathrm{C}$. Ten 
highly virulent, test isolates of $F$. udum, representing four agro-climatic zones were subjected to study for their cultural variability. Quantity of $20 \mathrm{ml}$ autoclaved and cooled PDA medium was dispensed in sterile glass petriplates (90 $\mathrm{mm}$ diam.) and allowed to solidify at room temperature. Aseptically, these plates were inoculated separately by putting in the centre a mycelial disc $(5 \mathrm{~mm})$ obtained from actively growing a week old pure culture of the test isolates and incubated at $27+1{ }^{\circ} \mathrm{C}$. Three PDA plates / isolate / replication were maintained

\section{Cultural variability}

Ten highly virulent, test isolates of $F$. udum, representing four agro-climatic zones were subjected to study for their cultural variability. The experiment was planned in CRD and the ten test isolates were replicated thrice.

Observations on cultural characteristics viz., colony diameter, colony colour, colony appearance, colony shape and colony margin, zonation, substrate pigmentation etc. were recorded after a week of incubation and sporulation was recorded at 10 days of incubation, sporulating culture of the test isolates in Petri plates was flooded with $10 \mathrm{ml}$ distilled water and was gently scraped with camel hair brush, to obtain spore suspension. Temporary mount on glass slide, of the spore suspension was prepared, mounted under research microscope (10X objective lens), counted the spores under five random microscopic fields and averaged. Based on (Kumar and Choudhary, 2006) scale, the test isolates were categorized.

\section{Morphological variability}

Temporary mounts in Lactophenol cotton blue stain on glass slides of the sporulated cultures of 10 test isolates were prepared separately and covered with glass slide. The morphological characteristics viz., length and breadth, septation of microconidia and macroconidia of each test isolate (10 days old pure culture growth on PDA) were recorded by using $\mathrm{J}$ image software, TS view and with the help of the compound microscope (make: Labomed Vision 2000 as well as Olympus) at 400X magnification under 10 random microscopic fields.

\section{Molecular variability}

Molecular variability among 10 isolates of $F$. udum was analyzed by RAPD molecular markers.

\section{Isolation of genomic DNA}

The genomic DNA of the 10 test isolates of $F$. udum was isolated, separately by using standard $2 \%$ cetyl trimethyl ammonium bromide (CTAB) extraction method.

\section{Quantification of DNA}

Spectrophotometer was used for quantitative and qualitative analysis of the DNA of the test isolates. Five $\mu 1$ of DNA sample was added in Cuvette carrying $0.995 \mu \mathrm{l}$ of sterile $\mathrm{H}_{2} \mathrm{O}$ and absorbance was measured at $280 \mathrm{~nm}$ wave lengths. Similarly, the purity of DNA was checked by measuring the ratio of OD at A260/A280 nm. The quantification of DNA was calculated by using following formula.

$\mathrm{OD}$ at $260 \mathrm{~nm} \mathrm{X}$ dilution factor

DNA $(\mu \mathrm{g} / \mu \mathrm{l})=$ - 1000

\section{Primer screening}

Available RAPD primers were used for screening of Fusarium udum. The primers were screened on the basis of reproducible and scorable amplification for analysis of Fusarium udum. For example, positively screened primers (OP series A to $\mathrm{Z}$ ) along with their sequence are mentioned below. 
Cultural variability

\begin{tabular}{|c|c|c|}
\hline Grade & Sporulation & No. of spores per microscopic field \\
\hline- & Absent & Nil \\
\hline+ & Poor & $1-10$ \\
\hline++ & Fair & $11-30$ \\
\hline+++ & Good & $31-50$ \\
\hline++++ & Excellent & More than 50 \\
\hline
\end{tabular}

Primer screening

\begin{tabular}{|c|c|c|c|c|c|}
\hline Sr. No. & Primer & Sequence 5'-3 & Sr. No. & Primer & \\
\hline 1 & OPA-03 & AGTCAGCCAC & 10 & OPC-05 & GATGACCGCC \\
\hline 2 & OPA-09 & GGGTAACGCC & 11 & OPC-14 & TGCGTGCTTG \\
\hline 3 & OPA-17 & GACCGCTTGT & 12 & OPC-19 & GTTGCCAGCC \\
\hline 4 & OPB-04 & GGACTGGAGT & 13 & OPC-20 & ACTTCGCCAC \\
\hline 5 & OPB-10 & CTGCTGGGAC & 14 & OPD-02 & GGACCCAACC \\
\hline 6 & OPB-12 & CCTTGACGCA & 15 & OPD-03 & GTCGCCGTCA \\
\hline 7 & OPB-15 & GGAGGGTGTT & 16 & OPD-05 & TGAGCGGACA \\
\hline 8 & OPB-20 & GGACCCTTAC & 17 & OPD-07 & TTGGCACGGG \\
\hline
\end{tabular}

RAPD analysis of $F$. udum isolates

The PCR protocol for RAPD reaction was optimized with various PCR components and thermal cycler programme. Master mix $(24 \mu \mathrm{l})$ containing all of the reactants, except template DNA were dispensed in autoclaved PCR tubes $(0.2 \mathrm{ml})$. Genomic DNA of each isolate of $F$. udum was added to the individual tubes containing the master mix.

The contents of each tube were mixed by tapping with fingers, followed by a brief spun to collect the content at bottom of the tube. These tubes were placed in Thermocycler (Bio Rad, USA) and subjected to PCR according to the standardized protocol.

The amplified RAPD product was separated by electrophoresis in $1.5 \%$ agarose gel with 1 $\mathrm{X}$ TAE buffer, stained with ethidium bromide $(0.5 \mu \mathrm{g} / \mathrm{ml})$ at $90 \mathrm{~V}$ for 1.0 to $1.5 \mathrm{hrs}$ and photographed using gel documentation system (Alpha Innotech, USA). The sizes of the amplification product were estimated using $100 \mathrm{bp}$ to $1 \mathrm{~kb}$ ladder (Fermentas, UK). The polymorphism was detected by comparing RAPD product of the test isolates of $F$. udum

\section{Data scoring and analysis}

The amplified products generated from RAPD-PCR reaction were resolved on $1.5 \%$ agarose gel. The RAPD amplicons showing monomorphic and polymorphic pattern were scored and amplicon size was determined by comparison with $1 \mathrm{~kb}$ DNA ladder (Fermentas, U.K.). Jaccard's similarity coefficient $(\mathrm{J})$ was used to calculate similarity between pairs of varieties, which was as follows (Jaccard, 1908).

$\mathrm{J}=\mathrm{n}_{\mathrm{xy}} / \mathrm{n}_{\mathrm{t}}-\mathrm{n}_{\mathrm{z}}$

$\mathrm{n}_{\mathrm{xy}}$ is the number of bands common to variety $\mathrm{x}$ and $\mathrm{y}$

$\mathrm{n}_{\mathrm{t}}$ is the total number of bands present in all samples and

$\mathrm{n}_{\mathrm{z}}$ is the number of bands absent in $\mathrm{x}$ and $\mathrm{y}$ but, found in all samples

RAPD fingerprint data was scored in present (1) or absent (0) forms, data matrices were generated and used to plot dendrogram exploited for phylogenetic analysis, by using Jacquards' similarity coefficient, using the software NTSYS pc2.02i and Exerter Software. 


\section{Results and Discussion}

Cultural variability among the $F$. udum isolates

The results obtained on cultural characteristics viz., mycelial growth (colony diameter), mycelial colour, colony appearance, growth speed, colony shape, margin, sporulation and pigmentation etc. in respect of 10 test isolates of F. udum grown on PDA (Table 1, 2 and Fig. 1).

\section{Mycelial growth}

The results indicated that among the test isolates, mycelial growth was varied from $54.67 \mathrm{~mm}$ (FOU 17) to $89 \mathrm{~mm}$ (FOU 16). However, it was the highest in isolate FOU 16 $(89 \mathrm{~mm})$, followed by the isolates viz., FOU $30(88.67 \mathrm{~mm})$, FOU $12(87.67 \mathrm{~mm})$, FOU 6 $(84.33 \mathrm{~mm})$, FOU $22(83.33 \mathrm{~mm})$, FOU 2 $(82.67 \mathrm{~mm})$ and these all six were at par with each other.

In rest of the test isolates, mycelial growth was ranged from $81.33 \mathrm{~mm}$ to $54.67 \mathrm{~mm}$. significantly; minimum mycelial growth was found in FOU 17 i.e. $54.67 \mathrm{~mm}$.

The maximum (> $80 \mathrm{~mm}$ ) colony diameter were seen in seven isolates i.e. FOU 2, FOU 6, FOU 12, FOU 16, FOU 19 and FOU 22 with $70 \%$ frequency and medium colony diameter was seen in isolate FOU 3 isolate with $10 \%$ frequency. However, minimum $(<60 \mathrm{~mm})$ colony diameter were seen in isolates FOU 13 and FOU 17 with $20 \%$ frequency.

\section{Colony colour}

On the basis of colony colour, the test isolates were categorized into four groups. Group I consisted three isolates with white colony (FOU 2, FOU 19 and FOU 30) shared $30 \%$ frequency. Group II contained two isolates with purple colony (FOU 3 and FOU 13) containing $20 \%$ frequency, group III consisted three isolates with pink colony (FOU 6, FOU 12 and FOU 17) had $30 \%$ frequency, group IV consisted two isolates with buff colony (FOU 16 and FOU 22) shared $20 \%$ frequency.

\section{Colony growth rate}

On the basis colony growth rate, the test isolates were categorized as fast growing, moderate growing and slow growing. In fast growing category, the five isolates viz., FOU 2, FOU 6, FOU 16, FOU 19 and FOU 30 were included with $50 \%$ frequency. In medium / moderate growing category, the two isolates viz., FOU 12 and FOU 22 were included with $20 \%$ frequency as well as in slow growing category the three isolates FOU 3, FOU 13 and FOU 17 were included with $30 \%$ frequency.

\section{Colony shape and margin}

On the basis of colony shape (circular) and colony margin (non-serrated, smoother serrated), the test isolates were categorized into two groups. The group I included two isolates with circular colony and non-serrated with smooth margin, which were FOU 19 and FOU 30 with $20 \%$ frequency. The group II included the isolates with circular colony and serrated margin, which contained rest of the eight isolates viz., FOU 2, FOU 3, FOU 6, FOU 12, FOU 13, FOU 16, FOU 17 and FOU 22 with $80 \%$ frequency.

\section{Sporulation and pigmentation}

The sporulation induced by the test isolates varied from fair $(++)$ to excellent $(++++)$. However, it was excellent (++++) in six isolates viz., FOU 2, FOU 6, FOU 12, FOU 13, FOU 16 and FOU 17 with $60 \%$ frequency; good (+++) in two isolates viz, FOU 3 and FOU 22 with $20 \%$ frequency and fair (++) in two isolates viz., FOU 19 and FOU 
30 with $20 \%$ frequency.

On the basis of pigmentation, the test isolates were categorized into five groups. The group I included one isolate with dark yellow pigmentation (FOU 2) with $10 \%$ frequency; the group II included four isolates with pink pigmentation (FOU 3, FOU 6, FOU 12 and FOU 13) with $40 \%$ frequency; the group III included two isolates with brown pigmentation (FOU 16 and FOU 22) with 20 $\%$ frequency.The group IV included one isolate with yellow to brown pigmentation (FOU 17) with $10 \%$ frequency and the group $\mathrm{V}$ included two isolates with light yellow pigmentation (FOU 19 and FOU 30) with 20 $\%$ frequency.

Table.1 Cultural variability among the test isolates of Fusarium udum

\begin{tabular}{|c|c|c|c|c|c|c|}
\hline \multirow{2}{*}{$\begin{array}{l}\text { Sr. } \\
\text { No. }\end{array}$} & \multirow[t]{2}{*}{ Parameters } & \multicolumn{5}{|c|}{ Isolates (District Location) / Characteristics } \\
\hline & & $\begin{array}{c}\text { FOU 2 } \\
\text { (Ahmednagar } \\
\text { ) }\end{array}$ & $\begin{array}{l}\text { FOU 3 } \\
\text { (Akola) }\end{array}$ & $\begin{array}{l}\text { FOU } 6 \\
\text { (Beed) }\end{array}$ & $\begin{array}{c}\text { FOU } 12 \\
\text { (Jalna- } \\
\text { Badnapur) }\end{array}$ & $\begin{array}{c}\text { FOU 13 } \\
\text { (Jalna- } \\
\text { Mantha) }\end{array}$ \\
\hline 1 & $\begin{array}{l}\text { Colony dia. } \\
(\mathrm{mm})\end{array}$ & 82.67 & 63.00 & 84.33 & 87.67 & 58.67 \\
\hline 2 & Colour & White & Purple & Pink & Pink & Purple \\
\hline 3 & $\begin{array}{l}\text { Mycelial } \\
\text { Appearance }\end{array}$ & $\begin{array}{l}\text { Luxuriant, } \\
\text { appressed, } \\
\text { felted and } \\
\text { fluffy }\end{array}$ & $\begin{array}{c}\text { Scanty, } \\
\text { partially } \\
\text { appressed and } \\
\text { fibrous }\end{array}$ & $\begin{array}{l}\text { Luxuriant, } \\
\text { appressed } \\
\text { and fluffy }\end{array}$ & $\begin{array}{l}\text { Luxuriant, } \\
\text { partially } \\
\text { appressed and } \\
\text { fibrous }\end{array}$ & $\begin{array}{c}\text { Scanty, } \\
\text { partially } \\
\text { appressed } \\
\text { and fibrous }\end{array}$ \\
\hline 4 & Growth speed & Fast & Slow & Fast & Medium & Slow \\
\hline 5 & Colony shape & Circular & Circular & Circular & Circular & Circular \\
\hline 6 & Colony margin & Serrated & Serrated & Serrated & Serrated & Serrated \\
\hline 7 & Sporulation & ++++ & +++ & ++++ & ++++ & ++++ \\
\hline 8 & Pigmentation & Dark Yellow & Pink & Pink & Pink & Pink \\
\hline
\end{tabular}

Continued....

\begin{tabular}{|c|c|c|c|c|c|c|}
\hline \multirow{2}{*}{$\begin{array}{l}\text { Sr. } \\
\text { No. }\end{array}$} & \multirow[t]{2}{*}{ Parameters } & \multicolumn{5}{|c|}{ Isolates (District Location) / Characteristics } \\
\hline & & $\begin{array}{l}\text { FOU } 16 \\
\text { (Latur) }\end{array}$ & $\begin{array}{c}\text { FOU } 17 \\
\text { (Nagpur) }\end{array}$ & $\begin{array}{l}\text { FOU } 19 \\
\text { (Nashik) }\end{array}$ & $\begin{array}{c}\text { FOU } 22 \\
\text { (Parbhani) }\end{array}$ & $\begin{array}{l}\text { FOU } 30 \\
\text { (Satara) }\end{array}$ \\
\hline 1 & Colony dia. (mm) & 89.00 & 54.67 & 81.33 & 83.33 & 88.67 \\
\hline 2 & Colour & Buff & Pink & White & Buff & White \\
\hline 3 & $\begin{array}{l}\text { Mycelial } \\
\text { Appearance }\end{array}$ & $\begin{array}{l}\text { Luxuriant, } \\
\text { partially } \\
\text { appressed and } \\
\text { fibrous }\end{array}$ & $\begin{array}{l}\text { Scanty, partially } \\
\text { appressed and } \\
\text { fibrous }\end{array}$ & $\begin{array}{l}\text { Luxuriant, } \\
\text { appressed, } \\
\text { felted and } \\
\text { fluffy }\end{array}$ & $\begin{array}{c}\text { Luxuriant, } \\
\text { partially } \\
\text { appressed and } \\
\text { fluffy }\end{array}$ & $\begin{array}{c}\text { Luxuriant, } \\
\text { appressed, } \\
\text { felted and } \\
\text { fluffy }\end{array}$ \\
\hline 4 & Growth speed & Fast & Slow & Fast & Medium & Fast \\
\hline 5 & Colony shape & Circular & Circular & Circular & Circular & Circular \\
\hline 6 & Colony margin & Serrated & Serrated & $\begin{array}{l}\text { Non-Serrated, } \\
\text { smooth }\end{array}$ & Serrated & $\begin{array}{l}\text { Non- } \\
\text { Serrated, } \\
\text { smooth }\end{array}$ \\
\hline 7 & Sporulation & ++++ & ++++ & ++ & +++ & ++ \\
\hline 8 & Pigmentation & Brown & Yellow to Brown & Light Yellow & Brown & $\begin{array}{l}\text { Light } \\
\text { Yellow }\end{array}$ \\
\hline
\end{tabular}

Sporulation: $++++=$ Excellent,$+++=$ Good,$++=$ Fair,$+=$ Poor, Dia: Diameter 
Table.2 Grouping and frequency of $F$. udum test isolates based on cultural variability

\begin{tabular}{|c|c|c|c|c|}
\hline Groups & Cultural parameters & $\begin{array}{l}\text { No. of } \\
\text { isolates }\end{array}$ & Isolates code & Frequency \\
\hline \multicolumn{5}{|c|}{ 1. Colony growth (Range and category) } \\
\hline Group-I & Maximum (> $80 \mathrm{~mm})$ & 07 & $\begin{array}{l}\text { FOU 2, 6, 12, 16, 19, } 22 \text { and } \\
30\end{array}$ & $70 \%$ \\
\hline Group-II & Medium (60 to $80 \mathrm{~mm}$ ) & 01 & FOU 3 & $10 \%$ \\
\hline Group-III & Minimum $(<60 \mathrm{~mm})$ & 02 & FOU 13 and FOU 17 & $20 \%$ \\
\hline \multicolumn{5}{|c|}{ 2. Colony colour } \\
\hline Group-I & White & 03 & FOU 2, FOU $19 \&$ FOU 30 & $30 \%$ \\
\hline Group-II & Purple & 02 & FOU 3 and FOU 13 & $20 \%$ \\
\hline Group-III & Pink & 03 & FOU 6, FOU $12 \&$ FOU 17 & $30 \%$ \\
\hline Group-IV & Buff & 02 & FOU 16 and FOU 22 & $20 \%$ \\
\hline \multicolumn{5}{|c|}{ 3. Mycelium appearance } \\
\hline Group I & Luxuriant and appressed & 04 & $\begin{array}{l}\text { FOU 2, FOU 6, FOU } 19 \text { and } \\
\text { FOU } 30\end{array}$ & $40 \%$ \\
\hline Group II & $\begin{array}{l}\text { Luxuriant and partially } \\
\text { appressed }\end{array}$ & 03 & FOU 12, FOU 16 \& FOU 22 & $30 \%$ \\
\hline Group III & $\begin{array}{l}\text { Scanty and partially } \\
\text { appressed }\end{array}$ & 03 & FOU 3, FOU $13 \&$ FOU 17 & $30 \%$ \\
\hline \multicolumn{5}{|c|}{ 4. Growth speed (mm / day) } \\
\hline Group I & Fast (12 mm / day) & 05 & FOU $2,6,16,19 \&$ FOU 30 & $50 \%$ \\
\hline Group II & Medium (10 mm / day) & 02 & FOU 12 and FOU 22 & $20 \%$ \\
\hline Group III & Slow (06 mm / day) & 03 & $\begin{array}{l}\text { FOU 3, FOU } 13 \text { and FOU } \\
17\end{array}$ & $30 \%$ \\
\hline \multicolumn{5}{|c|}{ 5. Colony margin } \\
\hline Group I & Serrated & 08 & $\begin{array}{l}\text { FOU 2, 3, 6, 12, 13, 16, } 17 \\
\text { and } 22\end{array}$ & $80 \%$ \\
\hline Group II & Non-serrated & 02 & FOU 19 and FOU 30 & $20 \%$ \\
\hline \multicolumn{5}{|c|}{ 6. Sporulation } \\
\hline Group I & Excellent $(++++)$ & 06 & $\begin{array}{l}\text { FOU 2, 6, 12,13,16 and } \\
\text { FOU } 17\end{array}$ & $60 \%$ \\
\hline Group II & Good $(+++)$ & 02 & FOU 3 and FOU 22 & $20 \%$ \\
\hline Group III & Fair $(++)$ & 02 & FOU 19 and FOU 30 & $20 \%$ \\
\hline \multicolumn{5}{|c|}{ 7. Pigmentation } \\
\hline Group I & Dark yellow & 01 & FOU 2 & $10 \%$ \\
\hline Group II & Light yellow & 02 & FOU 19 and FOU 30 & $20 \%$ \\
\hline Group III & Yellow to Brown & 01 & FOU 17 & $10 \%$ \\
\hline Group IV & Pink & 04 & $\begin{array}{l}\text { FOU 3, FOU 6, FOU } 12 \text { and } \\
\text { FOU } 13\end{array}$ & $40 \%$ \\
\hline Group V & Brown & 02 & FOU 16 and FOU 22 & $20 \%$ \\
\hline
\end{tabular}


Table.3 Morphological variability among the test isolates of $F$. udum

\begin{tabular}{|c|c|c|c|c|c|}
$\begin{array}{c}\text { Sr. } \\
\text { No. }\end{array}$ & Isolates & \multicolumn{2}{|c|}{ Micro-conidia } & \multicolumn{2}{c|}{ Macro-conidia } \\
\hline & & $\begin{array}{c}\text { Av. Size }(\boldsymbol{\mu m}) \\
\text { Length } \times \text { Breadth }\end{array}$ & $\begin{array}{c}\text { Septation } \\
(\mathbf{N o})\end{array}$ & $\begin{array}{c}\text { Av. Size }(\boldsymbol{\mu m}) \\
\text { Length } \times \text { Breadth }\end{array}$ & $\begin{array}{c}\text { Septation } \\
(\text { No. })\end{array}$ \\
\hline 1 & FOU 2 & $8.62 \times 3.70$ & 0 & $28.60 \times 4.30$ & $1-4$ \\
\hline 2 & FOU 3 & $7.41 \times 3.10$ & $0-1$ & $25.63 \times 4.40$ & $2-4$ \\
\hline 3 & FOU 6 & $6.55 \times 2.73$ & 0 & $23.20 \times 3.90$ & $1-3$ \\
\hline 4 & FOU 12 & $8.74 \times 3.92$ & 0 & $28.29 \times 4.10$ & $1-3$ \\
\hline 5 & FOU 13 & $6.32 \times 2.81$ & 0 & $26.22 \times 4.30$ & $2-4$ \\
\hline 6 & FOU 16 & $9.02 \times 4.10$ & 0 & $31.83 \times 3.89$ & $1-4$ \\
\hline 7 & FOU 17 & $9.34 \times 4.15$ & $0-1$ & $30.86 \times 5.52$ & $1-3$ \\
\hline 8 & FOU 19 & $5.92 \times 2.61$ & 0 & $22.40 \times 4.62$ & $2-3$ \\
\hline 9 & FOU 22 & $8.43 \times 3.54$ & 0 & $27.62 \times 4.10$ & $3-4$ \\
\hline 10 & FOU 30 & $5.33 \times 2.62$ & 0 & $24.80 \times 4.28$ & $1-2$ \\
\hline
\end{tabular}

Table.4 Grouping and frequency of $F$. udum test isolates based on morphological variability

\begin{tabular}{|c|c|c|c|c|c|}
\hline $\begin{array}{l}\text { Sr. } \\
\text { No. }\end{array}$ & Conidia & $\begin{array}{c}\text { Group and size } \\
(\mu \mathrm{m})\end{array}$ & $\begin{array}{l}\text { No. of } \\
\text { Isolates }\end{array}$ & Code of isolates & Frequency \\
\hline \multicolumn{6}{|c|}{ I. Length $x$ Breadth size } \\
\hline \multirow[t]{3}{*}{1} & \multirow{3}{*}{$\begin{array}{l}\text { Micro- } \\
\text { conidia }\end{array}$} & $\begin{array}{l}\text { Group I: Large } \\
(8.1-10 \mu \mathrm{m} \times 3-4 \mu \mathrm{m})\end{array}$ & 05 & $\begin{array}{c}\text { FOU } 2,12,16,17 \text { and } \\
\text { FOU } 22\end{array}$ & $50 \%$ \\
\hline & & $\begin{array}{l}\text { Group II: Medium } \\
(7.1-8 \mu \mathrm{m} \times 3-4 \mu \mathrm{m})\end{array}$ & 01 & FOU 3 & $10 \%$ \\
\hline & & $\begin{array}{l}\text { Group III: Small } \\
(5-7 \mu \mathrm{m} \times 2-3 \mu \mathrm{m})\end{array}$ & 04 & $\begin{array}{c}\text { FOU 6, } 13,19 \text { and FOU } \\
30\end{array}$ & $40 \%$ \\
\hline \multirow{3}{*}{2} & \multirow{3}{*}{$\begin{array}{l}\text { Macro- } \\
\text { conidia }\end{array}$} & $\begin{array}{l}\text { Group I: Large } \\
(28.1-32 \mu \mathrm{m} \times 4-6 \mu \mathrm{m})\end{array}$ & 04 & $\begin{array}{c}\text { FOU 2, 12, } 16 \text { and FOU } \\
17\end{array}$ & $40 \%$ \\
\hline & & $\begin{array}{l}\text { Group II: Medium } \\
(26.1-28 \mu \mathrm{m} \text { to } 4-5 \\
\mu \mathrm{m})\end{array}$ & 02 & FOU 13 \& FOU 22 & $20 \%$ \\
\hline & & $\begin{array}{l}\text { Group III: Small } \\
(22-26 \mu \mathrm{m} \times 3-5 \mu \mathrm{m})\end{array}$ & 04 & $\begin{array}{c}\text { FOU 3, 6, } 19 \text { and FOU } \\
30\end{array}$ & $40 \%$ \\
\hline \multicolumn{6}{|c|}{ II. Septation } \\
\hline \multirow[b]{2}{*}{1} & \multirow[b]{2}{*}{$\begin{array}{l}\text { Micro } \\
\text { conidia }\end{array}$} & $\begin{array}{l}\text { Group I: } \\
\text { No septation }\end{array}$ & 08 & $\begin{array}{c}\text { FOU } 2,6,12,13,16,19,22 \\
\text { and FOU } 30\end{array}$ & $80 \%$ \\
\hline & & $\begin{array}{l}\text { Group II: } \\
\text { Single septation }\end{array}$ & 02 & FOU 3 \& FOU 17 & $20 \%$ \\
\hline \multirow{3}{*}{2} & \multirow{3}{*}{$\begin{array}{l}\text { Macro } \\
\text { conidia }\end{array}$} & $\begin{array}{l}\text { Group I: Maximum } \\
(1-4,2-4 \& 3-4)\end{array}$ & 05 & $\begin{array}{c}\text { FOU } 2,3,13,16 \text { and } \\
\text { FOU } 22\end{array}$ & $50 \%$ \\
\hline & & $\begin{array}{l}\text { Group II: Medium } \\
(1-3, \& 2-3)\end{array}$ & 04 & $\begin{array}{c}\text { FOU 6, } 12,17 \text { and FOU } \\
19\end{array}$ & $40 \%$ \\
\hline & & $\begin{array}{l}\text { Group III: Minimum } \\
(1-2)\end{array}$ & 01 & FOU 30 & $10 \%$ \\
\hline
\end{tabular}


Table.5 Polymorphic amplifications generated by RAPD markers

\begin{tabular}{|c|l|c|c|c|c|c|}
\hline $\begin{array}{c}\text { Sr. } \\
\text { No. }\end{array}$ & Primer & $\begin{array}{c}\text { Total } \\
\text { No. of amplicons }\end{array}$ & $\begin{array}{c}\text { Average No. of } \\
\text { bands / primer }\end{array}$ & $\begin{array}{c}\text { Total No. } \\
\text { of Loci }\end{array}$ & $\begin{array}{c}\text { No. of polymorphic } \\
\text { Loci }\end{array}$ & $\begin{array}{c}\text { Per cent } \\
\text { Polymorphism (\%) }\end{array}$ \\
\hline 1. & OPA 9 & 10 & 0.1 & 3 & 3 & 100 \\
\hline 2. & OPA 17 & 7 & 0.7 & 4 & 4 & 100 \\
\hline 3. & OPA 3 & 9 & 0.9 & 4 & 4 & 100 \\
\hline 4. & OPB 4 & 19 & 1.9 & 5 & 5 & 100 \\
\hline 5. & OPB 12 & 12 & 1.2 & 4 & 4 & 100 \\
\hline 6. & OPB 15 & 15 & 1.5 & 9 & 9 & 100 \\
\hline 7. & OPC 1 & 9 & 0.9 & 2 & 2 & 100 \\
\hline 8. & OPC 5 & 6 & 0.6 & 3 & 3 & 100 \\
\hline 9. & OPC 14 & 18 & 1.8 & 7 & 7 & 100 \\
\hline 10. & OPD 2 & 19 & 1.9 & 7 & 7 & 100 \\
\hline 11. & OPD 3 & 23 & 2.3 & 6 & 6 & 100 \\
\hline 12. & OPD 5 & 16 & 1.6 & 8 & 8 & 100 \\
\hline 13. & OPD 7 & 14 & 1.4 & 5 & 5 & 100 \\
\hline 14. & OPC 19 & 21 & 2.1 & 8 & 8 & 100 \\
\hline 15. & OPC 20 & 16 & 1.6 & 7 & 7 & 100 \\
\hline 16. & OPB 10 & 38 & 3.8 & 10 & 10 & 100 \\
\hline 17. & OPB 20 & 22 & 2.2 & 6 & 6 & $\mathbf{1 0 0}$ \\
\hline Overall & $\mathbf{2 7 4}$ & - & $\mathbf{9 8}$ & $\mathbf{9 8}$ & $\mathbf{1 0 0}$ \\
\hline Average & $\mathbf{1 6 . 1 1}$ & $\mathbf{1 . 5 6}$ & $\mathbf{5 . 7 6}$ & $\mathbf{5 . 7 6}$ & \\
\hline
\end{tabular}

Table.6 Similarity index in DNA fingerprinting of $F$. udum isolates

\begin{tabular}{|c|c|c|c|c|c|c|c|c|c|c|}
\hline & FOU 2 & FOU 3 & FOU 6 & FOU 12 & FOU 13 & FOU 16 & FOU 17 & FOU 19 & FOU 22 & FOU 30 \\
\hline FOU 2 & 1.0000000 & & & & & & & & & \\
\hline FOU 3 & 0.0000000 & 1.0000000 & & & & & & & & \\
\hline FOU 6 & 0.0000000 & 0.1525424 & 1.0000000 & & & & & & & \\
\hline FOU 12 & 0.0000000 & 0.1363636 & 0.3846154 & 1.0000000 & & & & & & \\
\hline FOU 13 & 0.0000000 & 0.0882353 & 0.2553191 & 0.3666667 & 1.0000000 & & & & & \\
\hline FOU 16 & 0.1666667 & 0.0800000 & 0.0208333 & 0.0689655 & 0.0588235 & 1.0000000 & & & & \\
\hline FOU 17 & 0.0000000 & 0.2894737 & 0.1451613 & 0.1041667 & 0.1111111 & 0.0714286 & 1.0000000 & & & \\
\hline FOU 19 & 0.0000000 & 0.1600000 & 0.3793103 & 0.2916667 & 0.2250000 & 0.0540541 & 0.2200000 & 1.0000000 & & \\
\hline FOU 22 & 0.0217391 & 0.1964286 & 0.2714286 & 0.2241379 & 0.1372549 & 0.0909091 & 0.2500000 & 0.4629630 & 1.0000000 & \\
\hline FOU 30 & 0.0000000 & 0.2881356 & 0.3424658 & 0.2500000 & 0.1166667 & 0.0178571 & 0.2539683 & 0.3134328 & 0.2597403 & 1.0000000 \\
\hline
\end{tabular}

Table.7 Dis-similarity index in DNA fingerprinting of $F$. udum isolates

\begin{tabular}{|c|c|c|c|c|c|c|c|c|c|c|}
\hline & FOU 2 & FOU 3 & FOU 6 & FOU 12 & FOU 13 & FOU 16 & FOU 17 & FOU 19 & FOU 22 & FOU 30 \\
\hline FOU 2 & 0.0000000 & & & & & & & & & \\
\hline FOU 3 & 1.0000000 & 0.0000000 & & & & & & & & \\
\hline FOU 6 & 1.0000000 & 0.8474576 & 0.0000000 & & & & & & & \\
\hline FOU 12 & 1.0000000 & 0.8636364 & 0.6153846 & 0.0000000 & & & & & & \\
\hline FOU 13 & 1.0000000 & 0.9117647 & 0.7446809 & 0.6333333 & 0.0000000 & & & & & \\
\hline FOU 16 & 0.8333333 & 0.9200000 & 0.9791667 & 0.9310345 & 0.9411765 & 0.0000000 & & & & \\
\hline FOU 17 & 1.0000000 & 0.7105263 & 0.8548387 & 0.8958333 & 0.8888889 & 0.9285714 & 0.0000000 & & & \\
\hline FOU 19 & 1.0000000 & 0.8400000 & 0.6206897 & 0.7083333 & 0.7750000 & 0.9459459 & 0.7800000 & 0.0000000 & & \\
\hline FOU 22 & 0.9782609 & 0.8035714 & 0.7285714 & 0.7758621 & 0.8627451 & 0.9090909 & 0.7500000 & 0.5370370 & 0.0000000 & \\
\hline FOU 30 & 1.0000000 & 0.7118644 & 0.6575342 & 0.7500000 & 0.8833333 & 0.9821429 & 0.7460317 & 0.6865672 & 0.7402597 & 0.0000000 \\
\hline
\end{tabular}


Fig.1 Cultural (Colony growth) variability among the test isolates of $F$. udum

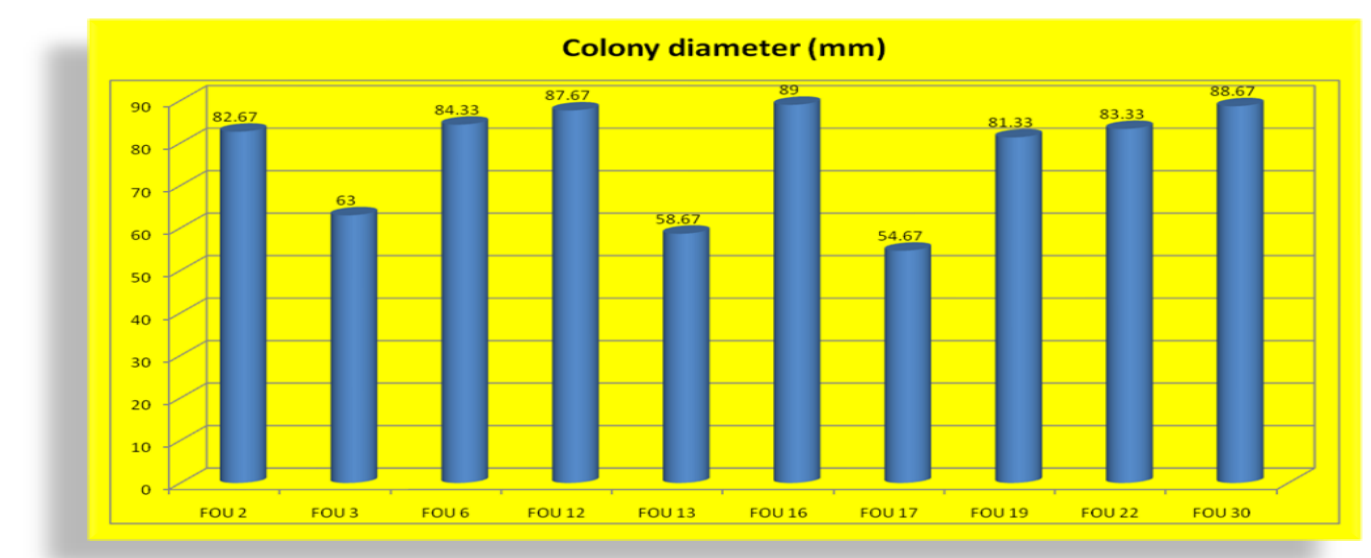

Fig.2 Dendrogram based on RAPD analysis depicting relationship between 10 test isolates of $F$. udum
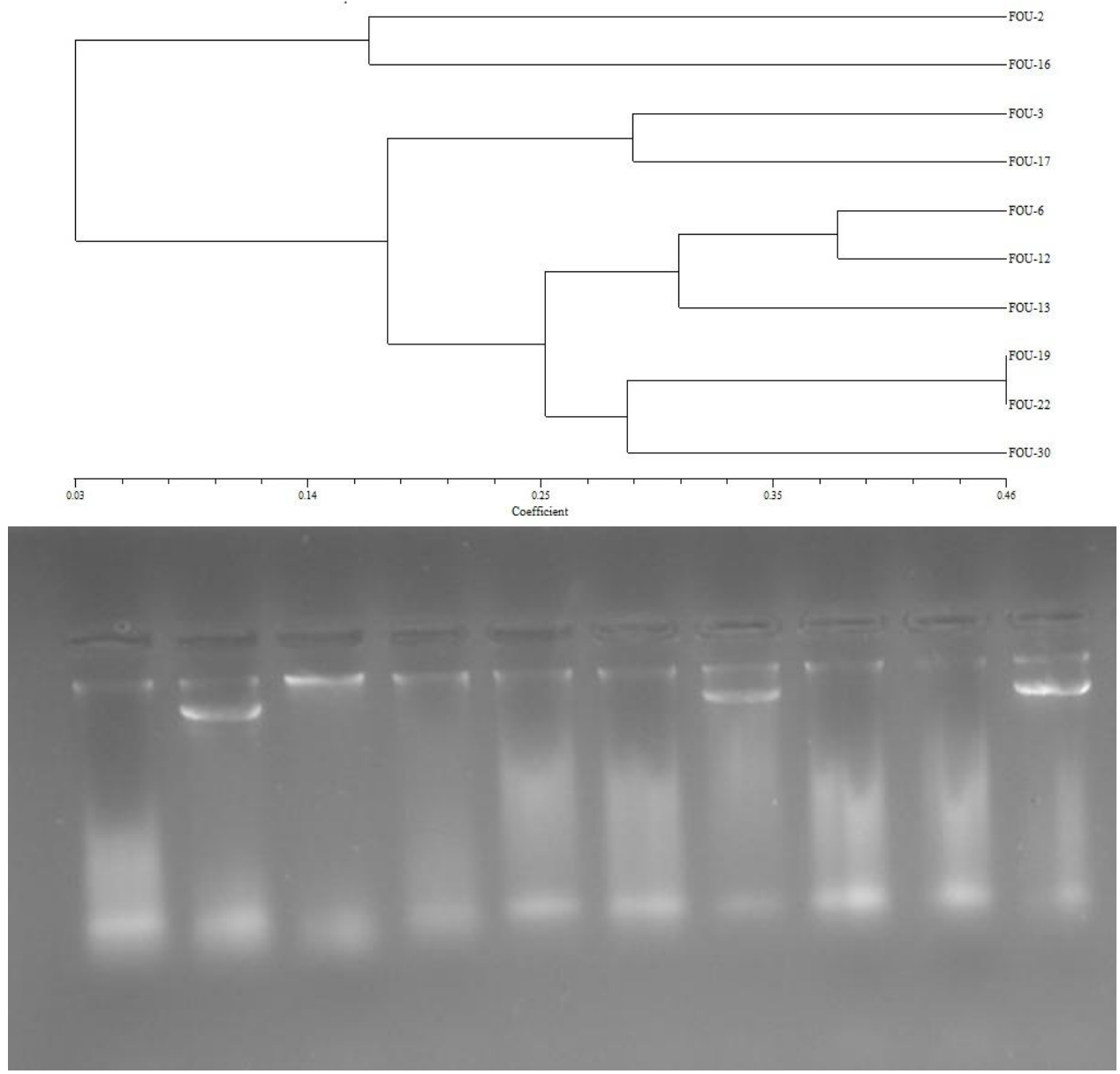

Fig.3 RAPD fingerprient profile of 10 isolates of $F$. udum (DNA) 

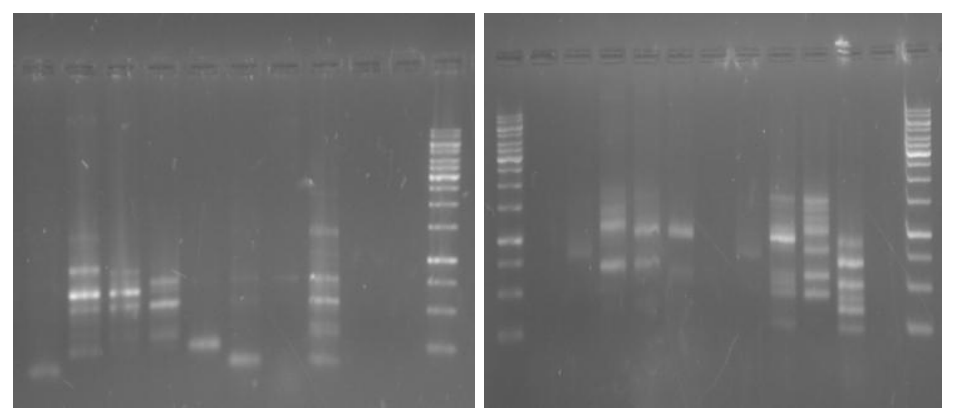

Fig.4 RAPD fingerprient profile of 10 isolates of $F$. udum by using primer OPC 19 \& OPC 20 Lane M- marker (1 kb DNA ladder); Lanes 1-10 isolates

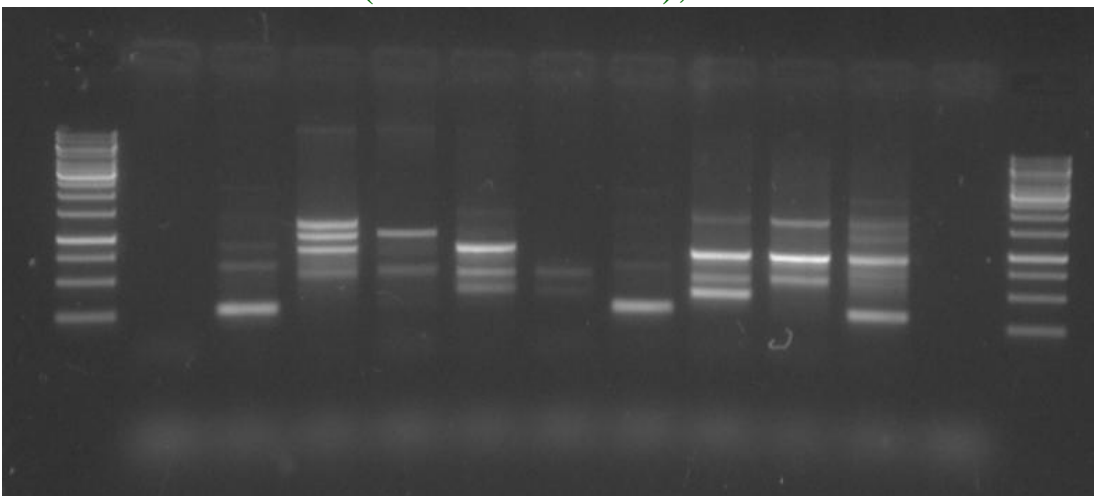

Fig. 5 RAPD fingerprient profile of 10 isolates of F. udum by using primer OPB 10 Lane Mmarker (1 kb DNA ladder); Lanes 1-10 isolates

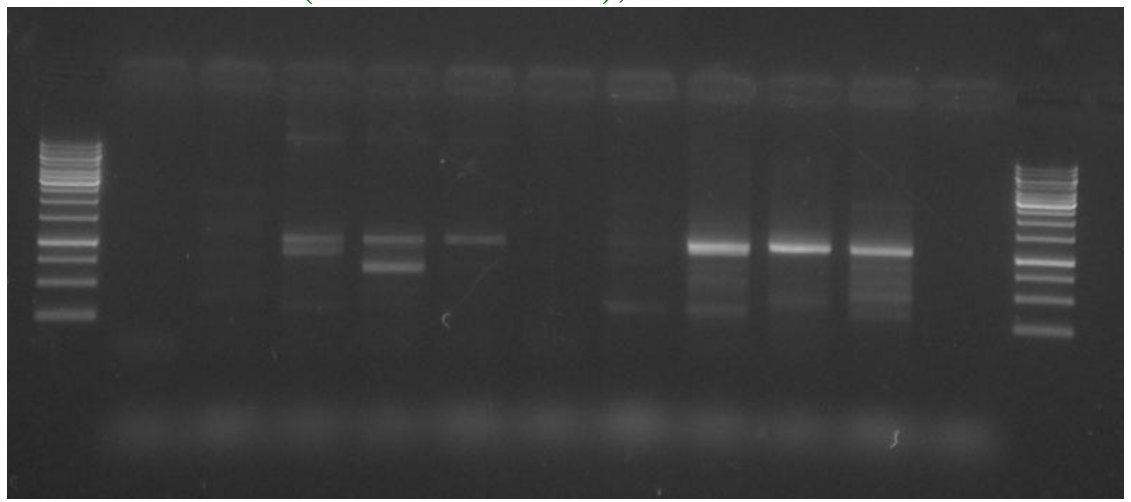

Fig.6 RAPD fingerprient profile of 10 isolates of $F$. udum by using primer OPB 20 Lane Mmarker (1 kb DNA ladder); Lanes 1-10 isolates

Morphological variability among $F$. udum isolates

\section{Conidial size}

The results revealed that all the ten isolates exhibited a wide range of variability in respect of size of microconidia and macroconidia. Average size of micro-conidia of the test isolates was ranged from $5.33 \mu \mathrm{m} \times 2.62$ $\mu \mathrm{m}$ (FOU 30) to $9.34 \mu \mathrm{m} \times 4.15 \mu \mathrm{m}$ (FOU 17). However, maximum micro-conidial size (9.34 x 4.15) was recorded in isolate FOU 17. This was followed by the isolates viz., FOU $16(9.02 \times 4.10)$, FOU $12(8.74 \times 3.92)$, FOU 2 (8.62 x 3.70), FOU $22(8.43 \times 3.54)$, FOU 3 (7.41 x 3.10), FOU 6 (6.55 x 2.73), FOU 13 $(6.32 \times 2.81)$, FOU $19(5.92 \times 2.61)$ and FOU $30(5.33 \mu \mathrm{m} \times 2.62 \mu \mathrm{m})$. 
Average size of macro-conidia of the test isolates was ranged from $22.40 \mu \mathrm{m} \times 4.62 \mu \mathrm{m}$ (FOU 19) to $31.83 \mu \mathrm{m} \times 3.89 \mu \mathrm{m}$ (FOU 16). However, maximum macro-conidial size (31.83 x 3.89) was recorded in isolate FOU 16.

This was followed by the isolates viz., FOU $17(30.86 \mu \mathrm{m} \times 5.52 \mu \mathrm{m})$, FOU $2(28.60 \times$ 4.30), FOU 12 (28.29 x 4.10), FOU 22 (27.62 $\mathrm{x} 4.10)$, FOU 13 (26.22 x 4.30), FOU 3 (25.63 $x$ 4.40), FOU $30(24.80 \times 4.28)$, FOU 6 (23.20 $\mathrm{x} 3.90)$ and FOU $19(22.40 \mu \mathrm{m}$ x $4.62 \mu \mathrm{m})$.

In case of micro-conidial size three groups viz., Group I (large), II (medium) and III (small) showed 50\%, $10 \%$ and $40 \%$ frequency respectively where as in case of macro-conidial size groups viz., Group I (large), II (medium) and III (small) showed $40 \%, 20 \%$ and $40 \%$ frequency, respectively.

\section{Septation}

Results revealed least variability was observed among the test isolates in respect of septa on the micro-conidia. Among the test isolates, septation was ranged from 0 to 1 . Isolates FOU 3 and FOU 17 were recorded 01 septation, whereas isolates FOU 2, FOU 6, FOU 12, FOU 13, FOU 16, FOU 19, FOU 22 and FOU 30 were more or less oval without septation.

Results revealed marked variability among the test isolates in respect of septa on the macro-conidia. Among the test isolates, septation was ranged from 1-2 to 3-4. Of the test isolates, FOU 22 recorded maximum (34) septation, followed by the isolates viz., FOU 3 and FOU 13 (2-4), FOU 19 (2-3), FOU 2 (1-4), FOU 6 as well as FOU 17 (1-3) and FOU 30 (1-2 septation).

In case of micro-conidial septation two groups viz., Group I (No septation) and II (single septation) showed $80 \%$ and $20 \%$ frequency, respectively. Whereas, in case of macro-conidial septation, three groups viz., Group I (maximum), II (medium) and III (minimum) showed $50 \%, 40 \%$ and $10 \%$ frequency, respectively. (Table 3 and 4)

The pathogenic, cultural and morphological variability of $F$. udum found in present study are in consonance with the earlier reports (Madhukeshwara and Seshadri, 2001; Kiprop et al., 2002; Reddy 2006; Mahesh et al., 2010; Tiwari and Dhar, 2011; Rangaswamy et al., 2012; Kumar and Upadhyay, 2014; Shinde et al., 2014, Rashmi and Chattannavar, 2016). These results indicated that there is existence of pathogenic, cultural and morphological variability in Fusarium oxysporum f. sp. udum which might be due to environmental variation or struggle of existence or such several causes.

\section{Molecular variability}

\section{DNA fingerprinting profile}

The RAPD-PCR protocol described by Chavan, (2004) was used with some modifications to produce DNA fingerprinting profile of 10 fungal isolates of $F$. udum species. The PCR amplification reaction was optimized by varying concentration of PCR components. Amplification reaction was carried out in $25 \mu \mathrm{l}$ reaction mixtures containing $30 \mathrm{ng}$ of fungal genomic DNA, 1X PCR buffer, $1.5 \mathrm{mM} \mathrm{MgCl}_{2}, 0.25 \mathrm{mM}$ dNTPs, 10 pmol primers and $1.50 \mathrm{U}$ of Taq DNA polymerase. PCR amplification was performed in master cycler gradient, Eppendorf PCR thermocycler.

The program consisted of an initial denaturing at $94{ }^{\circ} \mathrm{C}$ for $4 \mathrm{~min}$, followed by 39 cycles comprising denaturation at $94{ }^{\circ} \mathrm{C}, 1 \mathrm{~min}$, annealing at $37{ }^{\circ} \mathrm{C}$ and extension of $2 \mathrm{~min}$. at $72{ }^{\circ} \mathrm{C}$. The final extension was set at $72{ }^{\circ} \mathrm{C}$ for $10 \mathrm{~min}$. PCR amplified product was separated by electrophoresis on $1.5 \%$ agarose gel in $1 \mathrm{X}$ 
TAE buffer, stained with ethidium bromide and visualized under gel documentation system.

\section{Diversity analysis using RAPD marker}

The genomic DNA of 10 isolates of $F$. udum isolated from pigeonpea crop was subjected for PCR amplification by using RAPD primers. Initially 17 random primers viz., OPA to OPD series were screened (random primer kit A, Operon Tech., USA). These 17 primers were found more polymorphic and generated significant data for discrimination of the test 10 isolates.

The average size of amplicons generated by the test primers was ranged between $100 \mathrm{bp}$ to $10 \mathrm{~kb}$. The RAPD-PCR amplification results showed that about 17 RAPD primers generated a total of 274 bands, which were found polymorphic with an average of 16.11 bands per primer. The primers OPB-10, OPB15, OPC-19, OPC-5, OPC-14, OPD-02 and OPC-20 were found more informative, as they generated maximum number of bands i.e. 10, $9,8,8,7,7$ and 6 bands, respectively.

OPC-1, OPC-5 and OPA-9 generated low number of 2, 3 and 3 bands, respectively. All amplicons were found polymorphic with 100 $\%$ polymorphism. The similarity matrix based on Jaccard's coefficient was prepared by using the scored data of banding pattern with the help of NTSys pc software. The highest similarity (0.462) was found between the isolates FOU 19 and FOU 22 followed by 0.384 between FOU 6 and FOU 12. The lowest similarity $(0.00)$ was found between the isolates FOU 2 and FOU 30.

Dendrogram generated by UPGMA cluster analysis based on Jaccard's similarity coefficient obtained from RAPD markers through NTSys pc software revealed two major clusters. The first cluster consisted of two isolates namely FOU 2 and FOU 16 while second cluster contained eight isolates viz., FOU 3, FOU 17, FOU 6, FOU 12, FOU 13, FOU 19, FOU 22 and FOU 30. The isolates FOU 19 and FOU 22 were found to be most similar with $46 \%$ similarity. Exactly reciprocal / opposite results were obtained in dissimilarity index.

Molecular variability (Genetic diversity) among Fusarium udum was demonstrated earlier by many scientists. (Kumar et al., 2007; Datta et al., 2009; Kiprop et al., 2005; Prasad et al., 2012; Mesapogu et al., 2012 and Shinde et al., 2015)

Thus, in present study pathological, cultural, morphological and molecular variability observed among the isolates of $F$. udum may be attributed to their distribution in different Agro-climatic zones of the Maharashtra state, long term $F$. udum pathogen at a particular location and ability of the pathogens to adopt themselves in different cultivars (Table 5, 6, 7 and Fig. 2).

\section{References}

Anonymous (2017). Annual Report for 2017. Chief Statistician, Pune, Maharashtra, India.

Chavan, R. L. (2004). Study of genetic variability among isolates of Alternaria species infecting sunflower: cultural, morphological, pathological, biochemical and molecular investigations. M. Sc. (Agri.) Thesis, M.A.U. Parbhani., Pp. 1-72.

Dutta, S., Rita, R., Dhar, V., Chaudhary, R. G. and Gurha, S. N. (2009). RAPD based diagnosis and diversity analysis of Fusarium wilt pathogen of pulse crops. J. Food Legumes, 22 (2): 77-81.

Jaccard, P. (1908). Nawelle recherché surla distribution Florale. Bulletin de la Societc Vaucloise des Sciences Naturelles., 44: 223270.

Kannaiyan, J., Nene, Y. L., Reddy, M. V., Rayan, J. G. and Raju, T. N. (1984). Prevalence of pigeonpea diseases and associated crop losses 
in Asia and Amirica. Trop. J. Pest Management, 30: 62-71.

Kiprop, E. K., Baudoin, J. P., Mwang'ombe, A. W., Kimani, P. M. and Mergeai, G. (2002). Characterization of Kenyan Isolates of Fusarium udum from Pigeonpea [Cajanus cajan (L.) Millsp.] by Cultural Characteristics, Aggressiveness and AFLP Analysis., J. Phytopathol. 150 (10): 517-525.

Kiprop, E. K., Mwang'ombe, A. W., Baudoin, J. P., Kimani, P. M. and Mergeai, G. (2005). Genetic Variability among Fusarium udum isolates from pigeonpea. African J. Crop Sci., 13 (3): 163-172.

Kumar, D. and Choudhary, U. (2006). Influence of temperature on mycelial growth and sporulation of $A$. brassicae and $A$. brassicicola causing blight. J. Res. SKUASTJ. 5 (1): 48-51.

Kumar, S. and Upadhyay, J. P. (2014). Studies on cultural morphological and pathogenic variability in isolates of Fusarium udum causing wilt in pigeonpea. Indian Phytopathol, 67 (1): 55-58.

Kumar, V., Chavan, V. B. and Shrivastva, J. P. (2007). Pathogenic and biochemical variability in Fusarium udum causing pigeonpea wilt. Indian Phytopathol. 60 (3): 281-288.

Madhukeshwara, S. S. and Sesadri, V. S. (2001). Variation and management of Fusarium udum of pigeonpea (Cajanus cajan (L.) Millsp.) Trop. Agril. Res. 13: 380-394.

Mahesh, M., Saifulla, M., Prasad, P. S. and Sreenivasa, S. (2010). Studies on cultural variability of Fusarium udum isolates in India. Inter. J. Sci. Nature 1 (2): 219- 225.

Mesapogu, S., Bakshi, Achala, Babu, B. K., Reddy, S. S., Sexsena, S. and Arora, D. K. (2012). Genetic diversity and pathogenic variability among Indian isolates of Fusarium udum infecting pigeonpea (Cajanus cajan (L.) Millsp.). Inter. Research J. Agril. Sci. Soil Sci. 2 (1): 51-57.
Prasad, P. S., Saifulla, M., Mallikarjuna, N., Thimmegowda, P. R. and Lakshmipathy, R. N. (2012). Integrated disease management of Pigeonpea wilt Fusarium udum (Butler). Madras Agric. J., 99 (10): 811-814.

Rangaswany, E., Pushpavati, B., Mallikarjuna, M. G. and Reddy, P. N. (2012). Morphological and cultural characters of Fusarium udum. Bioinfolet, 9 (4): 572-575.

Rashmi, U. S. and Chattannavar, S. N. (2016). Cultural and morphological diversity among the isolates of Fusarium udum in Karnataka. J. Fa. M. Sci., 29 (23): 365-369.

Reddy, B. A. and Saifulla, M. (2006). Variation in growth and morphology of Fusarium udum isolates. Karnataka J. Agril. Sci., 19 (2): 318322.

Reddy, M. V., Nene, Y. L., Kannaiyan, J., Raju, T. N., Saka, V. N., Davor, A. T., Songa, W. P. and Omanga, P. (1990). Pigeonpea lines resistant to wilt in Kenya and Malawi. Inter. Pigeonpea Newsl, 6: 34.

Reddy, M. V., Raju, T. N, Sharma, S. B., Nene, Y. L. and McDonald, D. (1993). Hand book of pigeonpea diseases Information Bulletin. 42: 8-10.

Shinde, A. S., Kalaskar, S. R., Rathod, A. H., Sheikh, W. A. and Acharya, S. (2015). RAPD based molecular diversity analysis of different Fusarium udum Butler isolates of pigeonpea wilt. Inter.J. Pl. Protec., 8 (1): 81-85.

Shinde, V. S., Zagade, S. N. and Chavan, A. A. (2014). Cultural and morphological variation in Fusarium udum. J.Pl. Dis. Sci., 9 (2): 237244.

Tiwari, S. and Dhar, V. (2011). Prevalence of new variants of Fusarium udum in India. Indian Phytopath, 64: 243-246.

Upadhyay, R. S. and Rai, B., (1992). Wilt of pigeonpea. In: Plant Disease of International Importance (Eds. Singh, U.S., Mukhopadhyaya, A., Kumar, J. and Chaube, H.S.), Prentice Hall, Englewood Cliffs New Jersey, pp. 388-414.

\section{How to cite this article:}

Ghante, P.H., K.M. Kanase, S.P. Kale, R.L. Chavan, K.M. Sharma and Ghuge, S.B. 2018. Cultural, Morphological and Molecular Variability of Fusarium oxysporum f. sp. udum Isolates by RAPD Method. Int.J.Curr.Microbiol.App.Sci. 7(10): 2109-2122.

doi: https://doi.org/10.20546/ijcmas.2018.710.243 